\section{COVID-19 and gastrointestinal endoscopy in France: from the first to the second wave}

In March 2020, a week after the first lockdown in France, during the first wave of the SARS-CoV-2 pandemic, the French Society of Digestive Endoscopy (Société Française d'Endoscopie Digestive, SFED) sent gastroenterologists practicing endoscopy a web-based survey that included 35 questions about their endoscopic activity during 1 week [1]. The survey concluded that $91 \%$ of the endoscopies were cancelled, and underscored the poor level of personal protective equipment (PPE) available, with difficulties obtaining masks reported by $14 \%$ of respondents and $57 \%$ of endoscopies eventually performed without a mask. In addition, $13 \%$ of the respondents reported symptoms compatible with COVID-19 and $2 \%$ had a positive polymerase chain reaction (PCR) test for it.

The onset of a second wave of contaminations led the health authorities to declare a second lockdown, beginning on October 30, 2020. The SFED conducted a second survey, from November 23 , 2020 to November 27, 2020, to monitor changes in endoscopic practice, based on the lessons from the first wave. One hundred and twenty-four gastroenterologists took the survey, of whom, $61 \%$ were males, with a mean age of 50 years (range 29-69); 39\% worked in a public hospital. Ninety-six percent had read the SFED recommendations on endoscopy and COVID-19 [2]. However, only 63\% performed a routine SARS-CoV-2 PCR test on patients before endoscopy, 32\% wore a mask when performing endoscopies, and only $1 \%$ reported difficulty obtaining masks.

Sixty-nine percent of the healthcare facilities were involved in the care of patients with COVID-19, in $62 \%$ of centers, anesthesiologists were requisitioned, and $25 \%$ of the respondents were per- sonally involved in the care of patients with COVID-19 patients.

Twenty-one percent of the consultations were cancelled and $31 \%$ of the respondents performed consultations by telephone or video. Sixty-five percent of the respondents had to cancel endoscopies and 39\% of endoscopies were cancelled. During the week of the survey, the mean number of endoscopies performed on patients with COVID-19 was 4.6. Table 1 shows a comparison between data for the first and second wave.

Four percent of the respondents had positive SARS-CoV-2 serology. During the second wave, $12 \%$ of respondents reported symptoms compatible with COVID-19 and $4 \%$ had a positive PCR test.

In conclusion, the second wave of SARS-CoV-2 in France did not affect gastrointestinal endoscopy activity as significantly as the first wave. Uncertainty

- Table 1 Comparison of the impact of the first and second waves of the COVID-19 pandemic on gastrointestinal endoscopy activity in France.

\begin{tabular}{|c|c|c|c|}
\hline & First wave & Second wave & $P$ value \\
\hline Number of respondents to the survey & 694 & 124 & \\
\hline Patients with COVID-19 admitted to the hospital $-\mathrm{n}(\%)$ & $505 / 694(73 \%)$ & $86 / 124(69 \%)$ & 0.3 \\
\hline Anesthesiologists or specialized nurses requisitioned $-\mathrm{n}(\%)$ & $497 / 694(72 \%)$ & $77 / 124(62 \%)$ & 0.02 \\
\hline Involvement of gastroenterologists in management of patients with COVID-19 - n (\%) & $204 / 694(29 \%)$ & $31 / 124(25 \%)$ & 0.3 \\
\hline Proportion of cancelled consultations (\%) & $86 \%$ & $21 \%$ & 0.0001 \\
\hline Phone/video consultations $-\mathrm{n}(\%)$ & $398 / 694(57 \%)$ & $39 / 124(32 \%)$ & 0.0001 \\
\hline Endoscopists cancelling endoscopies - $\mathrm{n}(\%)$ & $685 / 694(99 \%)$ & $80 / 124(65 \%)$ & 0.0001 \\
\hline Proportion of cancelled endoscopies (\%) & $91 \%$ & $39 \%$ & 0.0001 \\
\hline Mean \pm SD number of endoscopic procedures for COVID-19 patients & $2.3 \pm 0.7$ & $4.6 \pm 4$ & 0.0001 \\
\hline Endoscopic procedures with a mask - $\mathrm{n}(\%)$ & $300 / 694(43 \%)$ & $40 / 124(32 \%)$ & 0.01 \\
\hline Difficulty obtaining surgical masks for endoscopy $-\mathrm{n}(\%)$ & $97 / 694(14 \%)$ & $1 / 124(1 \%)$ & 0.0001 \\
\hline \multicolumn{4}{|l|}{ COVID-19 infection among endoscopists } \\
\hline - COVID-19 symptoms - n (\%) & $89 / 694(13 \%)$ & $15 / 124(12 \%)$ & 0.88 \\
\hline - COVID-19 positive serology & - & $5 / 124(4 \%)$ & \\
\hline - COVID-19 infection documented by a positive PCR - n (\%) & $89 / 694(13 \%)$ & $5 / 124(4 \%)$ & 0.03 \\
\hline
\end{tabular}


about the duration of the pandemic and a need to avoid delayed diagnoses led to maintenance of scheduled gastrointestinal endoscopies during COVID-19 activity, as demonstrated by the lower proportion of consultation and endoscopies cancelled. Despite all the efforts made to release recommendations and the increase in availability of PPE, the level of protection of gastrointestinal endoscopists remained low, while the rate of contamination was stable between the first and second waves of the pandemic. This may be explained by the specific attention paid to keep SARS-CoV-2 out of endoscopy departments by checking all patients before endoscopy for absence of symptoms and negativity for SARS CoV-2 on PCR testing, and applying strict prevention methods in the few cases of endoscopy performed on patients with COVID-19. Finally, the results of this survey have several possible implications for the future. First, the practices of gastrointestinal endoscopists in terms of PPE seem difficult to change, and energy might be better spent on selecting patients before entering the endoscopy department (symptoms, PCR) and vaccinating patients and endoscopists. Second, the high (and stable) proportion of anesthesiologists requisitioned underscores the need for gastrointestinal endoscopists to gain autonomy, either by supervising the administration of sedation, improving the proportion of unsedated endoscopies, or using noninvasive endoscopic modalities, such as capsule endoscopy.

\section{Competing interests}

The authors declare that they have no conflict of interest.
The authors

Maximilien Barret ${ }^{1,2}$, Arthur Belle ${ }^{1}$, David Bernardini $^{3}$, Anne-Laure Tarrerias ${ }^{4}$, Erwan Bories $^{5}$, Vianna Costil ${ }^{6}$, Bernard Denis ${ }^{7}$, Rodica Gincul ${ }^{8}$, David Karsenti ${ }^{9}$, Stephane Koch $^{10}$, Arthur Laquiere ${ }^{11}$, Thierry Lecomte ${ }^{12}$, Vincent Quentin ${ }^{13}$, Gabriel Rahmi ${ }^{14}$, Michel Robaszkiewicz ${ }^{15}$, Eric Vaillant ${ }^{16}$, Geoffroy Vanbiervliet ${ }^{17}$, Arianne Vienne ${ }^{18}$, Franck Dumeiran $^{19}$, Olivier Gronier ${ }^{20}$, Stanislas Chaussade $^{1,2}$

1 Gastroenterology and Digestive Oncology, Cochin Hospital, Assistance PubliqueHôpitaux de Paris

2 University of Paris, France

3 Gastroenterology Department, La Casamance Private Hospital, Aubagne, France

4 Gastroenterology Department, Hôpital Foch, Suresnes, Île-de-France, France

5 Gastroenterology Department, Hôpital Privé de Provence, Aix En Provence, France

6 Gastroenterology Department, Pôle Santé des 4 Temps, Puteaux, France

7 Médecine A, Hôpital Pasteur, Colmar, France

8 Service de Gastroentérologie, Hôpital Edouard Herriot, Lyon, France

9 Digestive Endoscopy Unit, Clinique de Paris-Bercy, Charenton-le-Pont, France

10 Gastroenterology Department, $\mathrm{CHU}$ Besançon, Besançon, France

11 Gastroenterology Department, Hôpital St Joseph, Marseille, France

12 Department of Hepatogastroenterology and Digestive Oncology, Trousseau University Hospital, Tours, France

13 Digestive Endoscopy Unit, Hôpital St Brieuc, St Brieuc, France

14 Gastroenterology and Digestive Endoscopy, Georges Pompidou European Hospital, Paris, France

15 Department of Gastrointestinal Endoscopy, CHRU de Brest, Brest, France

16 Department of Gastroenterology, Centre médical du Nord, Lille, France

17 Digestive Endoscopy, Hôpital de L'Archet 2, Nice, France
18 Department of Gastroenterology, Hôpital privé d'Anthony, Anthony, France

19 Gastroenterology Department, Société française d'endoscopie digestive, Limoges, France

20 Department of Gastroenterology and Hepatology, Clinique Sainte Barbe, Strasbourg, France

Corresponding author

Maximilien Barret, MD, PhD

Gastroenterology and Digestive Oncology, Cochin Hospital, 27 rue du Faubourg St Jacques, 75014 Paris, France Fax: +0033158413865

maximilien.barret@aphp.fr

\section{References}

[1] Belle A, Barret M, Bernardini D et al. Impact of the COVID-19 pandemic on gastrointestinal endoscopy activity in France. Endoscopy 2020; 52: 1111-1115

[2] COVID-19 and endoscopy: Information and alerts for professionals. COVID-19 risk assessment and impact on digestive endoscopy management. French Society of Digestive Endoscopy; Accessed January 28, 2021 at: https://www.sfed.org/professionnels/covid19-et-endoscopie

\section{Bibliography}

Endosc Int Open 2021; 09: E1702-E1703

DOI 10.1055/a-1546-8665

ISSN 2364-3722

(C) 2021. The Author(s).

This is an open access article published by Thieme under the terms of the Creative Commons Attribution-NonDerivativeNonCommercial License, permitting copying and reproduction so long as the original work is given appropriate credit. Contents may not be used for commercial purposes, or adapted, remixed, transformed or built upon. (https:// creativecommons.org/licenses/by-nc-nd/4.0/)

Georg Thieme Verlag KG, Rüdigerstraße 14, 70469 Stuttgart, Germany 